\title{
THE OBSERVATIONAL SITUATION IN
}

\author{
REGARD TOBLACK HOLES
}

\author{
ROBERT E. WILSON \\ Dept. of Astronomy, University of South Florida, Fla., U.S.A. and Dept. of Physics and Astronomy, \\ University of Florida, Fla., U.S.A.
}

\begin{abstract}
Accumulated evidence on the existence of collapsed stars (black holes) in X-ray and non$\mathrm{X}$-ray binaries is considered. At present Cyg X-1 is the only strong case. The determinacy of solutions for mass ratios and other parameters of X-ray binaries is discussed, and some effects of tidal distortion and $\mathrm{X}$-ray heating on measured radial velocities and ellipsoidal light curves are described.
\end{abstract}

\section{Background}

Three main lines of reasoning have been used to argue that collapsed masses (black holes) are present in certain binary star systems. The first and least powerful of these might be called the gross underluminosity argument. It consists of a demonstration that one component of a binary is so extremely underluminous for its mass that it can be explained most simply in terms of a non-accreting black hole. Naturally, the underluminous mass must exceed the acceptable upper limit for low-luminosity equilibrium objects (degenerate dwarfs, neutron stars). Sometimes emphasis is placed on the degree of underluminosity in optical observations (the suspect in $\beta \mathrm{Lyr}$ is about six times more massive than the normal component, yet is invisible in the optical spectra) and sometimes on underluminosity over a large range of wavelength (in $\epsilon$ Aur the masses are comparable, but the secondary is quite invisible from the ultraviolet to the infrared). Of course other explanations are entirely possible, and the only claim is that these binaries should be suspected of harboring black holes. The second line of reasoning, which applies only to X-ray binaries, combines the above point about exceeding upper mass limits for degenerate stars with the argument that only objects whose ratio of mass to radius, $\left(M / M_{\odot}\right) /\left(R / R_{\odot}\right)$, is greater than about $10^{2}$ can realistically be expected to be X-ray sources in the commonly observed luminosity range of $10^{35}$ to $10^{38.5} \mathrm{erg} \mathrm{s}^{-1}$. A black hole is the only known type of object that would satisfy both the mass and (mass/radius) conditions, should a sufficiently massive X-ray source be found. To date, the only strong case in this category is Cyg X-1, although several lesser candidates have been recognized. Although this kind of evidence is at a much higher level of significance than the gross under-luminosity argument, one must admit that it, too, can only point to suspected black holes. The third approach is potentially very powerful. It consists of modelling the rapid variations in X-ray flux for the inner accretion disk around a black hole, and comparing the results with high-timeresolution X-ray observations. Since the most rapid variations should arise from the innermost orbits (highest temperature regions) the Fourier spectrum of the variations will be a function of X-ray energy, and a very large amount of information should be present in the observations. However, in order to take full advantage of these possibilities for fingerprinting black holes, work on the properties of accretion disks around compact objects (viz. Shakura, 1973; Shakura and Sunyaev, 1973; Lightman and Eardley, 1974; Lightman, 1974a, 1974b; Lightman and Shapiro, 1975; Pringle and Rees, 1972; Pringle et al., 1973) will require considerable development. However for Cyg X-1, 
Rothschild et al. (1974) have observed X-ray millisecond variations (including bursts), and this is the right time scale for the periods of the innermost orbits around a $10 M_{\odot}$ black hole.

If one takes the optimistic view that it is only a matter of time until the millisecond variation approach makes possible positive identifications of black holes, one might then ask why so much effort should be expended investigating the other kinds of information available from X-ray binaries. However, in order to understand the evolution of these systems, we must obtain accurate information of types not given by studies of accretion disks. We should know the mass, radius, luminosity, state of rotation, and other characteristics of the optical companion. To this end we have, for example, the observed variations with orbital phase of X-ray and optical flux, and of orbital velocity, but the interpretations of these observations in terms of tides, heating effects, and eclipses are not so straightforward as for normal binaries, so some of these problems will be discussed in Section 3 .

\section{Black Holes in X-Ray Binaries?}

In numerous papers (e.g. Bolton, 1972; Hutchings et al., 1973a; Hensberge and van den Heuvel, 1974; Mauder, 1973; Paczynski, 1974; Avni and Bahcall, 1975; Lyutyi et al., $1973,1974)$ it has been shown that it is virtually impossible to avoid finding a mass for Cyg X-1 considerably in excess of the theoretical upper limits $\left(\approx 1\right.$ to $\left.2 M_{\odot}\right)$ for uniformly-rotating degenerate dwarfs and neutron stars. As a necessary step in these arguments, one must have a reliable estimate of the distance, but this is now established at about $2.5 \pm 0.4 \mathrm{kpc}$ (Margon et al., 1973; Bregman et al., 1973) by means of color excess studies in the field of Cyg X-1. Given the spectroscopic mass function, $f(m)=M_{x}^{3}$ $\sin ^{3} i /\left(M_{\mathrm{o}}+M_{x}\right)^{2} \approx 0.2$, the inclination and one additional mass quantity are needed to fix $M_{x}$. For Cyg X-1, this information has been inferred from:

(a) the ellipsoidal variation of the optical star (Mauder, 1973; Avni and Bahcall, 1975);

(b) the assumption that $M_{\mathrm{o}}$ is normal for the observed spectral type and luminosity class (Bolton, 1972; Mauder, 1973);

(c) the surface gravity as derived from the optical spectra (Mauder, 1973);

(d) a combination of the constraint that the optical star not exceed its Roche lobe with a determination of the absolute radius by means of the observed luminosity and the known emissivity of stellar surfaces (Paczynski, 1974).

The last of these methods seems the most reliable since the underlying assumptions are quite minimal, and it specifically does not depend on ellipsoidal variation, for which different observers have found different amplitudes (Walker and Quintanilla, 1974; Lester et al., 1973) or on $\log g$, which is difficult to estimate to sufficient accuracy. Method (d), in fact, is not materially different from that used by Huang (1962), by Woolf (1965) and by Wilson (1974) for $\beta$ Lyr, and it should prove useful for other X-ray binaries. For a distance of $2.0 \mathrm{kpc}$, Paczynski (1974) finds $M_{x} \geqslant 6.4 M_{\odot}$, and for $2.5 \mathrm{kpc}$ he finds $M_{x} \geqslant 9.5 M_{\odot}$. Of course, both of these values well exceed the currently estimated limits for degenerate star masses. Although methods (a), (b), and (c) seem less well founded than (d), they also produce minimum values for $M_{x}$ considerably over the degenerate star limits. If we recall also the observed millisecond $\mathrm{X}$-ray flux variations, we must say that at present there is strong evidence for, and no important evidence against a black hole in Cyg X-1. Space does not permit discussion of several attempts which have been made to 
circumvent the [mass, mass/radius] argument by means of, for example, a triple star system (Bahcall et al., 1974) or non-accretion generation of X-rays (Bahcall et al., 1973). However the continued appearance of such models, even if they are subsequently ruled out, shows that the [mass, mass/radius] argument cannot be expected to prove the existence of a black hole.

Table I lists six X-ray binaries and one radio binary for which some discussion of possible black hole components has appeared, each with a short summary of the evidence or lack of evidence.

\section{TABLE I}

Black hole candidates

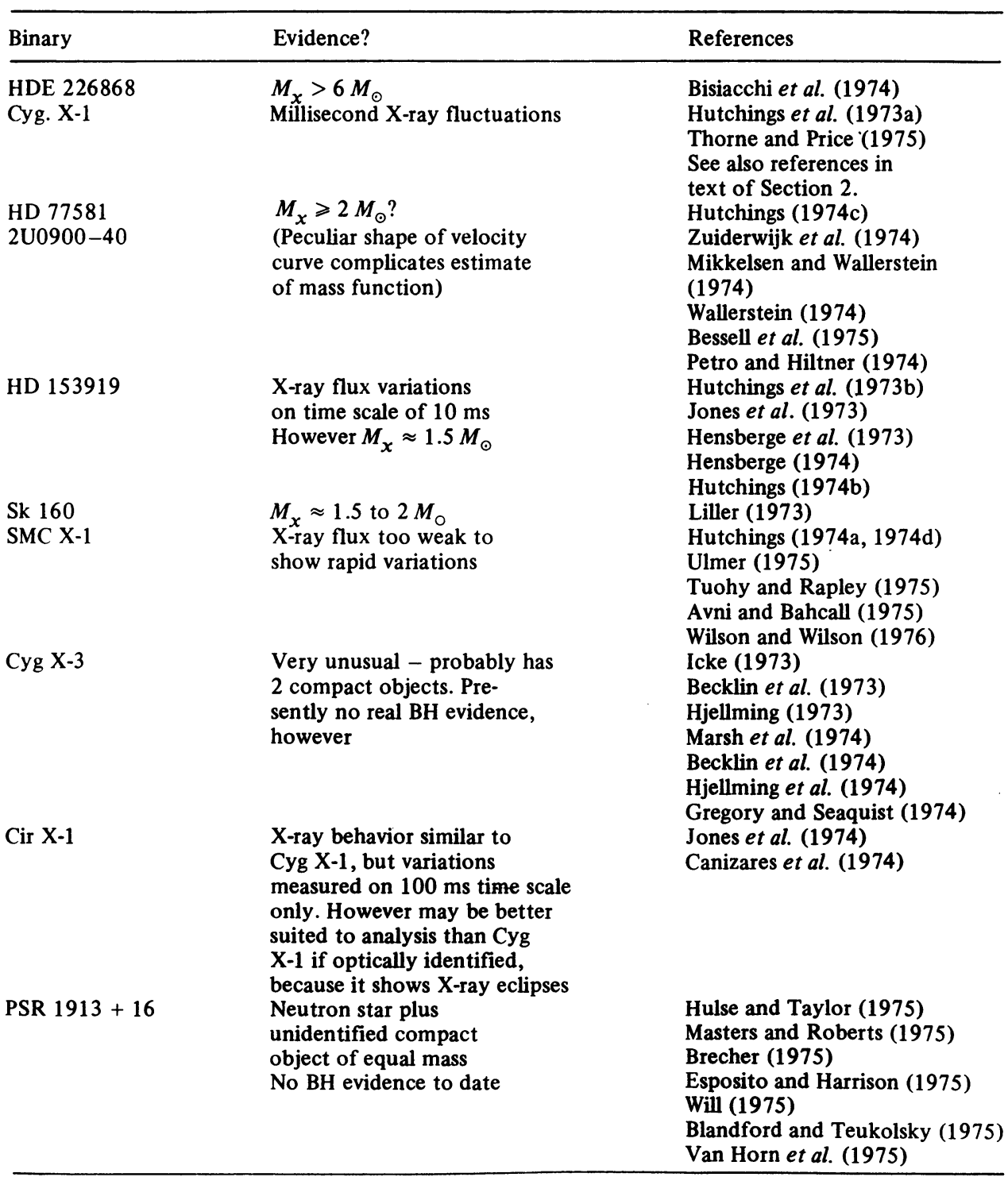




\section{Determinacy and Perturbation Effects}

Considerable efforts have been made (Hutchings 1974a,c,d; Avni and Bahcall, 1975; Whelan, 1973; Wilson and Wilson, 1976; many others) to extract information on masses, radii and luminosities of the components of $\mathrm{X}$-ray binaries from the observations - i.e. magnitudes, colors, light curves, radial velocity curves and X-ray eclipses. Here we consider two basic questions that naturally arise in this context. Firstly, given that the Roche model is applicable (and since most papers on this subject include at least some discussion of the effects of departures from the Roche model, these will not be considered here), is there sufficient information in the observations to yield unique values for the main parameters of the problem? Secondly, even within the context of the Roche model, are there effects that are not accounted for in most present-day work?

A simple basis for deciding the first issue has been given by Wilson and Wilson (1976). They point out that, since Fourier cosine terms higher than the $2 \theta$ term are essentially negligible in the theories of both ellipsoidal variation and the reflection effect (and sine terms are absent), one can express the brightness variation in terms of two relations in four unknowns. These are

$$
E 1+R 1=f 1\left(i, q, R_{0} / R_{\text {Roche }}, L_{0} / L_{\mathbf{x}}\right),
$$

and

$$
E 2+R 2=f 2\left(i, q, R_{0} / R_{\text {Roche }}, L_{0} / L_{\mathbf{x}}\right),
$$

where $E 1, E 2, R 1$, and $R 2$ are the $1 \theta$ and $2 \theta$ Fourier cosine coefficients for ellipsoidal variation $(E)$ and reflection $(R), i$ is orbital inclination, $q$ is mass ratio $\left(M_{x} / M_{\mathrm{o}}\right), R_{0}$ and $R_{\text {Roche }}$ are the radii of the optical star and its Roche lobe, and $L_{0}$ and $L_{x}$ are the luminosities of the optical and X-ray components, respectively. A third relation is available from the duration, $D$, of the $\mathrm{X}$-ray eclipse,

$$
D=f 3\left(i, q, R_{0} / R_{\text {Roche }}\right) \text {. }
$$

Thus, if we neglect the very small information carried by the $3 \theta, 4 \theta$, etc. terms, the general problem has no unique solution ( 3 relations, 4 unknowns). However special cases may be soluble, such as that in which it is virtually certain that $L_{x}$ is negligible compared to $L_{0}$, or in which $q$ is known from some other type of observation. Furthermore, as we shall see below, ellipsoidal variation for the very small values of $q$ found for some X-ray binaries has greater harmonic content than that of normal cases, so that $E 3$ and $E 4$ may not be negligible. Some numerical results [not including relation (3)] on the indeterminacy discussed here will appear in a paper by Wilson and Sofia (1976a) on HD 153919 (2U 1700-37).

On the second issue (new effects), Wilson and Sofia (1976b) call attention to the fact that a radial velocity effect predicted by Sterne (1941), but never actually observed, would be important for many X-ray binaries because it becomes large, relative to the orbital velocity, for small values of $q$. The effect arises because the observed velocity is, in effect, a weighted mean of the velocities of surface elements and not the true centerof-mass velocity of the star. This weighted mean is affected by geometric and photometric effects on a tidally distorted, irradiated star, and is shown for several cases in Figures 1 and 2. It seems likely at present that the peculiar velocity curve of HD 77581 (2U 0900-40) (Hutchings, 1974c; Zuiderwijk et al., 1974; Wallerstein, 1974), is due to 


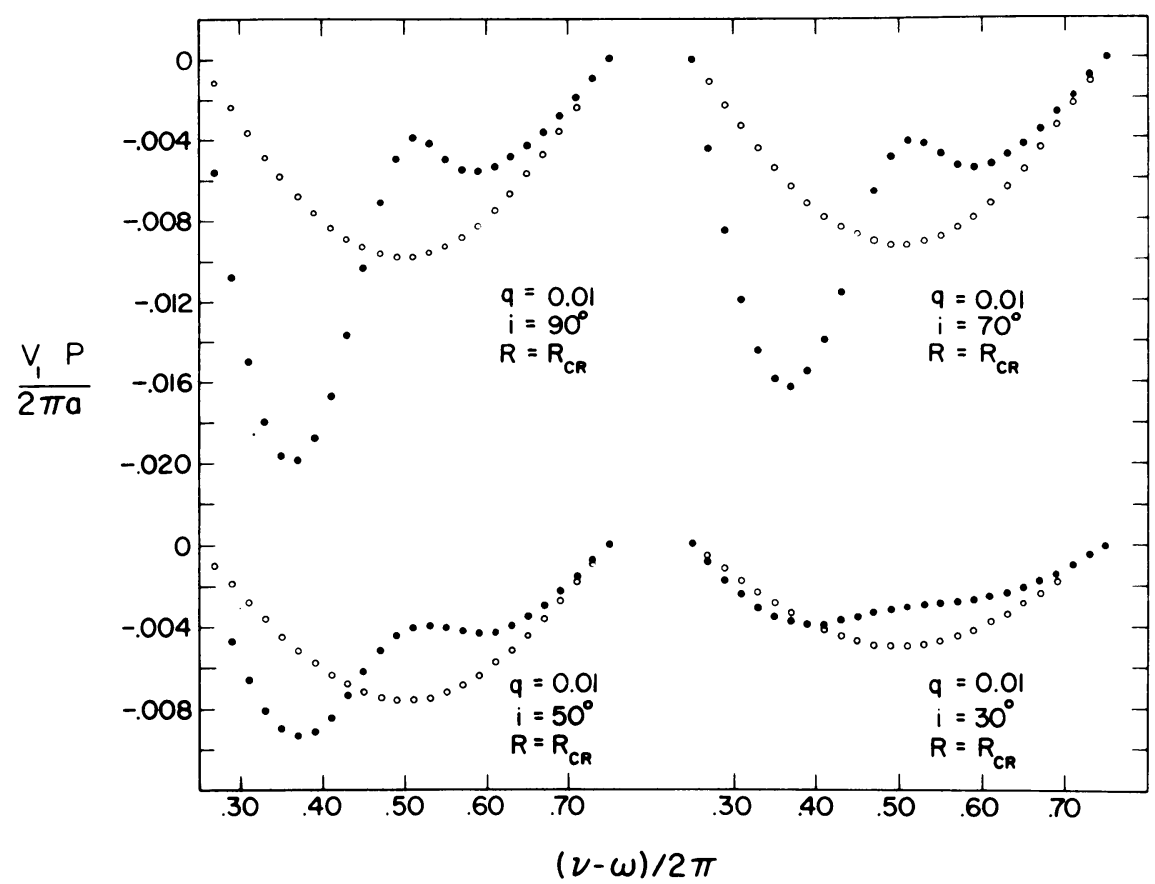

Fig. 1. Dimensionless radial velocity curves for four cases of extreme mass ratio, showing both the orbital velocity (open circles) and the directly measurable mean surface velocity (filled circles) for the optical components of X-ray binary systems. $R_{\mathrm{cr}}$ is the critical radius for filling the Roche lobe, and $q$ is $M_{x} / M_{\odot}$. Only half the phase range is shown, since the curves are antisymmetric about spectroscopic phase 0.25 .

this effect, in combination with a small orbital eccentricity. The effect would also be significant for Sk 160 (SMC X-1) as shown by Figure 3, although the large scatter makes it difficult to recognize the expected departures in the observations. For Sk 160, X-ray heating is definitely significant, so Figure 3 shows the combined effects of tidal distortion and the reflection effect as well as that due to tidal distortion alone.

Figure 4, which also is taken from Wilson and Sofia (1976b), shows the effects of extreme mass ratio on the form of ellipsoidal light curves. Notice that the curves are quite distinctly different from normal ellipsoidal cosine curves. This, of course, means that they have niore harmonic content than those for normal mass ratios, and thus contain more information about the binary system. Naturally, in principle this circumstance can relieve the indeterminacy discussed earlier and may, perhaps, render it possible to obtain unique solutions for system parameters in some favorable (small $q$ ) cases.

Although it might seem, at first sight, that these effects at small $q$ are troublesome complications on the analysis, they actually provide valuable information about the system which is not available for cases with large $q$.

\section{Black Holes in Non-X-Ray Binaries?}

Only two binaries that are not X-ray sources have attracted much discussion about pos- 


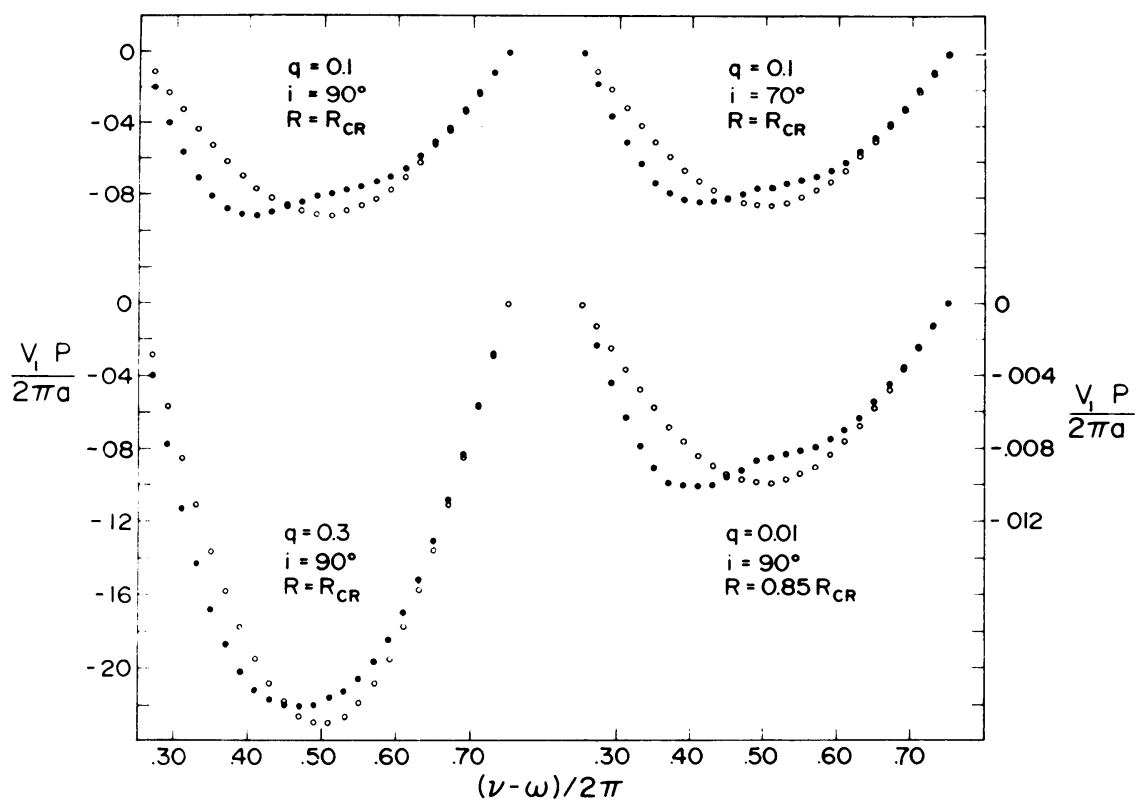

Fig. 2. Dimensionless radial velocity curves for four more cases, three with less extreme $q$ than in Figure 1, and one for a star only $85 \%$ as large as its Roche lobe.

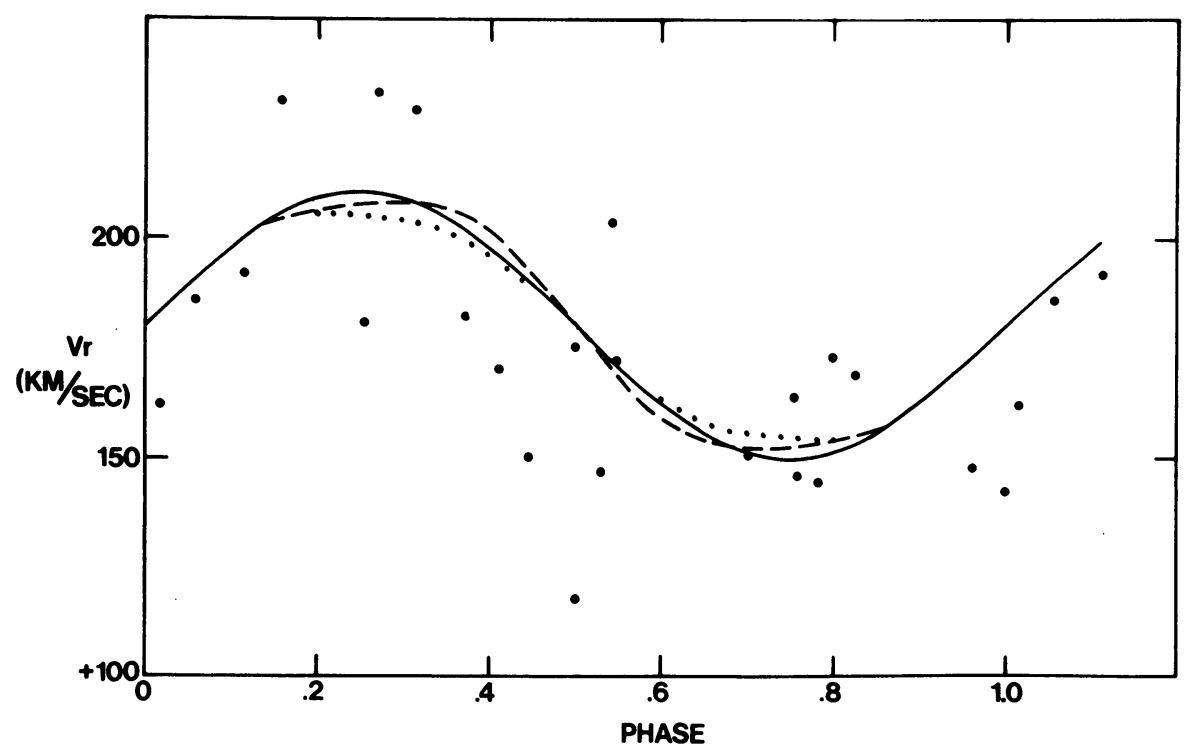

Fig. 3. Graph to show the size of the effects of Figures 1 and 2 for Sk 160 (SMC X-1). The continuous line represents the orbital velocity, the dashed line the combined effect of orbital velocity plus tidal distortion, and the dotted line shows orbital velocity plus tidal distortion plus the effects of X-ray heating. 


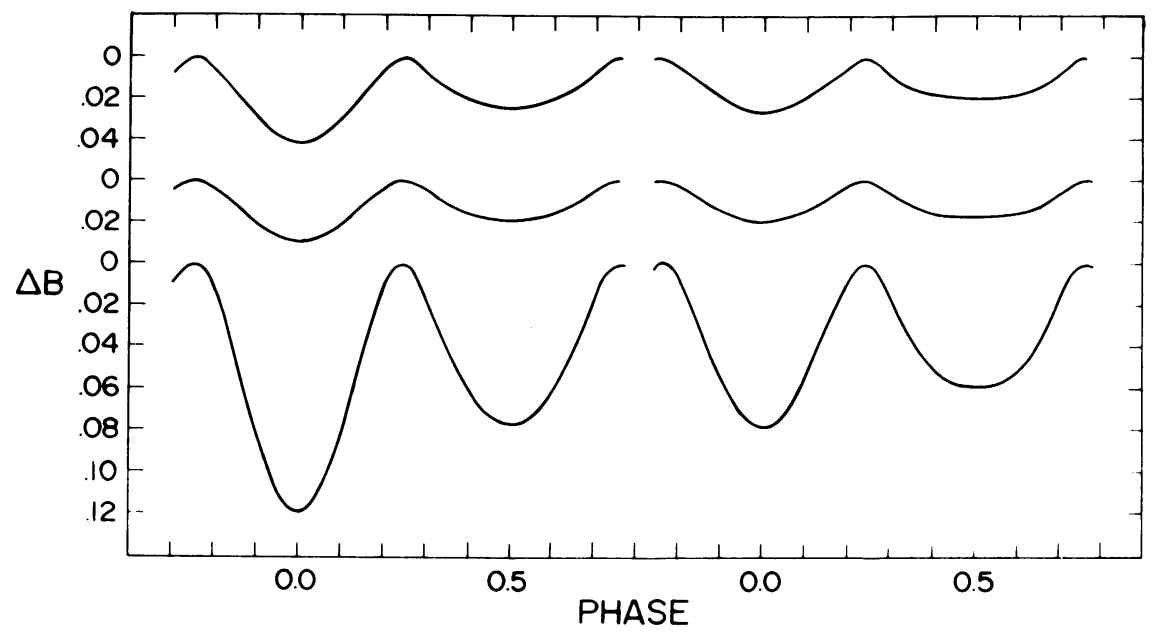

Fig. 4. Ellipsoidal variation for extreme mass ratio. Note the strong departure from sinusoids. Curves on the left include gravity darkening while those on the right do not. The lowermost curves have mass ratio 0.10 while the upper four have mass ratio 0.01 . The middle curves have $i=60^{\circ}$. All others have $i=90^{\circ}$. Effective wavelength is $4400 \AA$.

sible black holes. These are $\epsilon$ Aur (Cameron, 1971; Stothers, 1971; Wilson, 1971a) and $\beta$ Lyr (Devinney, 1971; Wilson, 1971b; Kondo et al., 1971; Wilson, 1974; Kriz, 1973, 1974; Ziołkowski, 1973; Hack et al., 1975). BMOri was proposed by Wilson (1972), but Popper and Plavec (1975) have now observed the secondary spectrum, which is that of late A-type star - probably the differentially rotating star proposed by Hall (1971). Some other cases (e.g. $\delta \mathrm{Gem}$, SV Cen, $\beta$ Per) have lost brief candidacies, including even demotion from binary star status for $\delta$ Gem (Trimble and Thorne, 1969; Abt and Levy, 1974; Tremaine et al., 1974).

Although nothing new has happened in the case of $\epsilon$ Aur since 1971, that of $\beta$ Lyr is relatively active, since several recent papers against a black hole, and one in favor of a black hole, have appeared. The original argument by Devinney (1971) was of the gross underluminosity type. Indeed, there does appear to be a very large underluminosity for the photometric secondary since it is now quite clear that it is the primary as judged by mass, and by a large margin. However Stothers (1972) pointed out that a considerable part of this apparent underluminosity is due to the fact that the primary is at an evolutionary stage at which it is very overluminous, so that the secondary looks underluminous by comparison. Then it was suggested by Kriz $(1973,1974)$ and shown quantitatively by Wilson (1974) that the remaining underluminosity is not real, but due to a combination of effects which are known to operate in $\beta$ Lyr. Also Ziołkowski $(1973,1975)$ has succeeded in reproducing the present configuration by entirely normal binary-star evolution computations with conservative mass transfer. Thus there is no difficulty in understanding $\beta$ Lyr as a straightforward example of the first epoch of rapid mass transfer, with the photometric secondary (the hypothetical black hole) being a main sequence star in the center of an opaque circumstellar disk of recently transferred material. Our understanding of the essential features of this system, which began with the Huang (1963) disk model, 
now seems quite solid. However Hack et al. (1975) again raised the issue of a $\beta$ Lyr black hole for a new reason. They state that PCyg lines of NV, observed in ultraviolet spectra taken by the Copernicus satellite, are stronger in $\beta$ Lyr than in any other star, even those of type 05. Since one component of $\beta$ Lyr is only of type B9 II while the other, based on its mass, would be about $\mathrm{BO} \mathrm{V}$ if the normal interpretations are accepted, Hack et al. (1975) suggest that the energetics for producing such strongly ionized nitrogen might be associated in some way with a black hole. However, the following counter-argument seems quite relevant in this context. First, we note that, in practice, the strengths of emission lines are normally estimated relative to the continuum. Second, the continuum in $\beta \mathrm{Lyr}$ is enormously reduced below that which it should have, because the more massive and more luminous star is hidden from the observer within a geometrically and optically thick disk. Thus any NV lines that may be present will appear anomalously strong by comparison with the reduced continuum radiation. It would be worthwhile to pursue this point quantitatively, but at present the $\mathrm{NV}$ lines would not seem to provide strong support for a black hole. Since, as mentioned above, the original underluminosity argument no longer exists because the secondary luminosity is quite normal, and since a massive black hole buried in a thick disk should actually be greatly overluminous for its mass (due to accretion luminosity) relative to a main sequence star, it seems improbable at present that the $\beta$ Lyr secondary is a black hole.

For $\epsilon$ Aur, the first argument for a black hole secondary component by Cameron (1971 - see also Stothers, 1971) was of the gross underluminosity type. To explain the flat-bottomed eclipses of $\epsilon$ Aur, Cameron's model involved a large disk, around the secondary, which was untenable on dynamical grounds because its plane was nearly normal to the orbit plane. Wilson (1971a) then proposed a disk model similar to the rings of Saturn, inclined only about one degree to the orbit plane. However, even this small inclination, which is necessary to produce eclipses of the observed depth, has been criticized for dynamical reasons. The problem is that the secondary mass must have axial symmetry (i.e. be oblate) and not spherical symmetry in order to maintain a thin disk even slightly inclined to the orbit. Therefore if the thin ring model is correct, the secondary mass cannot be a black hole, because the gravitational field of a black hole is spherically symmetric at large distances from the hole. Alternatively, if the thin ring model is not correct, the only viable alternative is the thick disk model by Huang (1965), which does not in any way require a black hole. In summary, there now seem to be no active candidates for black holes in optical binaries.

\section{References}

Abt, H. A. and Levy, S. G.: 1974, Astrophys. J. 188, 291.

Avni, Y. and Bahcall, J. N.: 1975, Astrophys. J. 197, 675.

Bahcall, J. N., Dyson, F. J., Katz, J. I., and Paczynski, B.: 1974, Astrophys. J. 189, L17.

Bahcall, J. N., Rosenbluth, M. N., and Kulsrud, R. M.: 1973, Nature Phys. Sci. $243,27$.

Becklin, E. E., Neugebauer, G., Hawkins, F. J., Mason, K. O., Sanford, P. W., Matthews, K., and Wynn-Williams, C. G.: 1973, Nature 245, 302.

Becklin, E. E., Hawkins, F. J., Mason, K. O., Matthews, K., Neugebauer, G., Packman, D., Sanford, P. W., Schulper, B., Stark, A., and Wynn-Williams, C. G.: 1974, Astrophys. J. 192, 119.

Bessell, M. S., Vidal, N. V., and Wickramasinghe, D. T.: 1975, Astrophys. J. 195, L117.

Bisiacchi, G. F., Dultzin, D., Firmani, C., and Hacyan, S.: 1974, Astrophys. J. 190, L59.

Blandford, R. and Teukolsky, S. A.: 1975, Astrophys. J. 198, L27.

Bolton, C. T.: 1972, Nature Phys. Sci. 240, 124. 
Brecher, K.: 1975, Astrophys. J. 195, L113.

Bregman, J., Butler, D., Kemper, E., Koski, A., Kraft, R. P., and Stone, R. P. S.: 1973, Astrophys. J. $185, \mathrm{~L} 117$.

Cameron, A. G. W.: 1971, Nature 229, 178.

Canizares, C. R., Li, F. K., and Clark, G. W.: 1974, Astrophys. J. 191, L75.

Devinney, E. J.: 1971, Nature 223, 110.

Esposito, L. W., and Harrison, E. R.: 1975, Astrophys. J. 196, L1.

Gregory, P. C. and Seaquist, E. R.: 1974, Astrophys. J. 194, 715.

Hack, M., Hutchings, J. B., Kondo, Y., McCluskey, G. E., Plavec, M., and Polidan, R. S.: 1975, Astrophys. J. 198, 453 .

Hall, D. S.: 1971, IAU Colloq. 15, 217.

Hensberge, G. and Heuvel, E. P. J. van den: 1974, Astron. Astrophys. 33, 311.

Hensberge, G., Heuvel, E. P. J. van den, and Paes dos Barros, M. H.: 1973, Astron. Astrophys. 29, 69.

Hensberge, G.: 1974, Astron. Astrophys. 36, 295.

Hjellming, R. M.: 1973, Science 182, 1089.

Hjellming, R. M., Brown, R. L., and Blankenship, L. C.: 1974, Astrophys. J. 194, L13.

Huang, S.: 1962, Astrophys. J. 136, 903.

Huang, S.: 1963, Astrophys. J. 138, 342.

Huang, S.: 1965, Astrophys. J. 141, 976.

Hulse, R. A. and Taylor, J. H.: 1975, Astrophys. J. 195, L51.

Hutchings, J. B.: 1974a, Astrophys. J. 188, 341.

Hutchings, J. B.: 1974b, Astrophys. J. 192, 677.

Hutchings, J. B.: 1974c, Astrophys. J. 192, 685.

Hutchings, J. B.: 1974d, Astrophys. J. 193, L61.

Hutchings, J. B., Crampton, D., Glaspey, J., and Walker, G. A. H.: 1973a, Astrophys. J. 182, 549.

Hutchings, J. B., Thackeray, A. D., Webster, B. L., and Andrews, P. J.: 1973b, Monthly Notices Roy. Astron. Soc. 163, 13P.

Icke, V.: 1973, Nature Phys. Sci. 244, 132.

Jones, C., Forman, W., Tananbaum, H., Schreier, E., Gursky, H., Kellogg, E., and Giacconi, R.: 1973, Astrophys. J. 181, L43.

Jones, C., Giacconi, R., Forman, W., and Tananbaum, H.: 1974, Astrophys. J. 191, L71.

Kondo, Y., McCluskey, G. E., and Houck, T. E.: 1971, IAU Colloq. 15, 308.

Kriz, S.: 1973, Nature 245, 36.

Kriz, S.: 1974, Bull. Astron. Inst. Czech. 25, 6.

Lester, D. F., Nolt, I. G., and Radostitz, J. V.: 1973, Nature 241, 125.

Lightman, A. P.: 1974a, Astrophys. J. 194, 419.

Lightman, A. P.: 1974b, Astrophys. J. 194, 429.

Lightman, A. P. and Eardley, D. M.: 1974, Astrophys. J. 187, L1.

Lightman, A. P. and Shapiro, S. L.: 1975, Astrophys. J. 198, L73.

Liller, W.: 1973, IAU Circ. No. 2469.

Lyutyi, V. M., Sunyaev, R. A., and Cherepaschuk, A. M.: 1973, Soviet Astron. - AJ 17,1 (transl. from Astron. Zh. 50, 3).

Lyutyi, V. M., Sunyaev, R. A., and Cherepaschuk, A. M.: 1974, Astron. Zh. 51, 1150.

Margon, B., Bowyer, S., and Stone, R. P. S.: 1973, Astrophys. J. 185, L113.

Marsh, K. A., Purton, C. R., and Feldman, P. A.: 1974, Astrophys. J. 192, 697.

Masters, A. R. and Roberts, D. H.: 1975, Astrophys. J. 195, L107.

Mauder, H.: 1973, Astron. Astrophys. 28, 473.

Mikkelsen, D. R. and Wallerstein, G.: 1974, Astrophys. J. 194, 459.

Paczynski, B.: 1974, Astron. Astrophys. 34, 161.

Petro, L. D. and Hiltner, W. A.: 1974. Astrophys. J. 190, 661.

Popper, D. M. and Plavec, M. M.: 1975, Astrophys. J. 205, 462.

Pringle, J. E. and Rees, M. J.: 1972, Astron. Astrophys. 21, 1.

Pringle, J. E., Rees, M. J., and Pacholczyk, A. G.: 1973, Astron. Astrophys. 29, 179.

Rothschild, R. E., Boldt, E. A., Holt, S. S., and Serlemitsos, P. J.: 1974, Astrophys. J. 189, L13.

Shakura, N. I.: 1973, Soviet Astron. - AJ 16, 756. (transl. from Astron. Zh. 49, 921).

Shakura, N. I. and Sunyaev, R. A.: 1973, Astron. Astrophys. 24, 337.

Sterne, T. E.: 1941, Proc. Natl. Acad. Sci. (U.S.) 27, 168.

Stothers, R.: 1971, Nature 229, 180.

Stothers, R.: 1972, Nature 238, 5.

Thorne, K. S. and Price, R. H.: 1975, Astrophys. J. 195, L101. 
Tremaine, S. D., Groth, E. J., and Nelson, M. R.: 1974, Astron. J. 79, 649.

Trimble, V. L. and Thorne, K. S.: 1969, Astrophys. J. 156, 1013.

Tuohy, I. R. and Rapley, C. G.: 1975, Astrophys. J. 198, L69.

Ulmer, M. P.: 1975, Astrophys. J. 196, 827.

Van Horn, H. M., Sofia, S., Savedoff, M. P., Duffy, J. E., and Berg, R. A.: 1975, Science 188, 930.

Walker, E. N. and Quintanilla, A. R.: 1974, Monthly Notices Roy. Astron. Soc. 169, 247.

Wallerstein, G.: 1974, Astrophys. J. 194, 451.

Whelan, J.: 1973, Astrophys. J. 185, L127.

Will, C. M.: 1975, Astrophys. J. 196, L3.

Wilson, R. E.: 1971a, Astrophys. J. 170, 529.

Wilson, R. E.: 1971b, Nature 234, 406.

Wilson, R. E.: 1972, Astrophys. Space Sci. 19, 165.

Wilson, R. E.: 1974, Astrophys. J. 189, 319.

Wilson, R. E. and Sofia, S.: 1976a (to be published).

Wilson, R. E. and Sofia, S.: 1976b, Astrophys. J. 203, 182.

Wilson, R. E. and Wilson, A. T.: 1976, Astrophys. J. 204, 551.

Woolf, N. J.: 1965, Astrophys. J. 141, 155.

Ziołkowski, J.: 1973, Bull. Am. Astron. Soc. 6, 263.

Ziołkowski, J.: 1975, Astrophys. J. 204, 512.

Zuiderwijk, E. J., Heuvel, E. P. J. van den, and Hensberge, G.: 1974, Astron. Astrophys. 35, 353.

\section{DISCUSSION}

Hutchings: The $\mathrm{N} V$ lines in $\beta$ Lyr have broad profiles and are maximal at quadratures. These suggest that the lines arise in the disk and preferentially between the stars. This has to be explained by any model for the system.

Wilson: But how could the nitrogen be ionized by the black hole if the $\mathrm{N} V$ lines originate between the stars?

Gursky: I would like to comment on your statement that 2U 0900-40 is another example of a pulsing X-ray source. This refers to the recent result from SAS-C which reports a periodicity of $284 \mathrm{~s}$ in the X-ray emission. However the effect is seen only when the source is very bright (which is not frequently the case) and the duty cycle is not very great $(\sim 20 \%)$. Thus this behaviour appears to be very different from the $\mathrm{X}$-ray pulsing observed in Cen X-3 or Her X-1 and may represent a totally different phenomenon.

Wu: This is similar to the behaviour of dwarf novae which also show optical pulses with period of tens of seconds only during the flaring state.

Van den Heuvel: According to the pictures of the pulses of 2U 0900-40 obtained with the SAS-C satellite the pulse has a lot of fine structure, which repeats from period to period. There is, among other things, a clear subpulse. Further, the X-ray spectrum is very hard, which suggests a neutron star rather than a white dwarf.

Plavec: Concerning the object SMC X-1, if you accept the two mutually agreeing solutions you get an even smaller mass, i.e. a very undermassive $O$ star.

Wilson: Yes, but I don't accept them. The most important numbers in the table of SMC X-1 parameters are the probable errors, which show that quite a large range of mass ratio is permitted, even by the purely internal errors.

Whelan: D. Wickramasinghe and I have made an extensive study of the SMCX1-Sk160 X-ray binary. We have used, as additional constraints, membership of SMC and X-ray heating effects. We find that these constraints are more useful than those imposed by radial velocity spectroscopy. The details are published elsewhere.

Bolton: There are dangers in using some of the observed light curves of the X-ray binaries. I have found that the various unpublished and published light curves have not been reduced to the same $U B V$ system so that there are great external inconsistencies among them even though they are internally consistent. There is a great need to get a standard set of comparison stars for these objects and to get them well tied in to the $U B V$ system.

Rucinski: In all light synthesis solutions one should include the uncertainty due to the adoption of the light level between minima equal to unity (or of a given magnitude as the reference level); this does not usually influence the solution but it does influence the errors (they might become, say, two times larger!). Did you include such a correction in your least-squares process?

Wilson: The value of the normalizing factor had been included as a free parameter in my calculations. This was done by including $L_{1}$ and $L_{2}$ as separate parameters, which include the scaling factor. 